\title{
Evaluation of web applications based on UX parameters
}

\author{
Anish Mistry, R. Arokia Paul Rajan \\ Department of Computer Science, CHRIST (Deemed to be University), India
}

\begin{tabular}{l} 
Article Info \\
\hline Article history: \\
Received Sep 30, 2018 \\
Revised Dec 11, 2018 \\
Accepted Mar 4, 2019 \\
\hline Keywords: \\
HCI \\
Responsive web \\
User experience \\
UX parameters \\
Web 2.0
\end{tabular}

\begin{abstract}
The objective of evaluating User Experience (UX) in this era of technology is to enhance the user satisfaction. Earlier applications were built with the aim of reducing the work of users. But with the evolution of the technology, the emergence of new gadgets and new trends in the information technology, the applications had to be more user-centric. The primary objective of this research is to evaluate the user experience of web applications based on different UX parameters using different techniques and given a rating. Each of these ratings are combined to determine the overall rating of UX for the web application. Also, the secondary objective of this research is to provide suggestions or recommendations based on the ratings to improve the UX of the web applications. An experimental study was conducted and the results show a significant improvement. Areas of further enhancements have also been identified and presented.
\end{abstract}

Copyright $@ 2019$ Institute of Advanced Engineering and Science. All rights reserved.

\section{Corresponding Author:}

Anish Mistry,

Department of Computer Science,

CHRIST (Deemed to be University),

Bengaluru, Karnataka - 560 029, India.

Email: mistry.nitinbhai@mca.christuniversity.in

\section{INTRODUCTION}

The term "User Experience" or "UX" is used widely but interpreted comprehensively. The multidisciplinary idea of UX has provoked a couple of implications of and perspectives on UX, each pushing towards the thought from a distinguishing viewpoint [1]. Existing denotations for UX go from cognitive to a business perspective and from quality driven to value driven. As Hassenzal and Tractinsky assert that the notion of UX aims at exceeding the shortcomings of Human Computer Interaction (HCI) by highlighting pertinent factors such as attractiveness, pleasure, satisfaction and individual advancement that fulfil traditional human requirements but have little importance. UX evaluation is comparatively simpler with precedent applications that individuals are found to be using as a part of their daily lives for a while. It is all the more difficult to assess UX prior to when it is simply a model or even an idea on the paper. All things considered we have to take in mind that every application will permit the proposed experiments before it is out for people to use. The prior we can assess UX, the more probable will be the effectiveness of the application [2].

Be that as it may, there are a few challenges to overcome. The initial introduction is frequently altogether different from the continuing UX, yet we can't run about the continuing field with ideas that don't work for all the things considered. We can't assess UX in the genuine settings when the framework is only a thought on the paper. Also, we can't perceive by what means the distinctive components of the framework will make a comprehensive UX when we have a thought of one new component as it were. It is difficult to run repetitive evaluations while the intended clients are on a separate mainland in comparison to the improvement. It might be hard to concur with a shared objective with the outer partners who impact the UX [3]. As of late, HCI scientists have started investigating the relationship amongst client and item such as its emotional characteristics as opposed to effectiveness; encounters instead of execution; fun and playability instead of fault rate; and amiability and emotional characteristics as opposed to intelligence. 
In this manner, we locate that viable and intense UX assessing methods are equipped for advancing over long stretches of time and it is basic to discover or create the fitting and develop methods for the collecting and investigating exact information in connection with these fresh, well-versed and significant sense of HCI [4].

There are many different ways and techniques to evaluate UX of web applications. This arises a question about the choice of technique to be used. Selecting an appropriate method can turn out to be challenging because it may vary based on different requirements and context of where it is being used. These techniques can be subjective, quantifiable, automated or manual. Most of these techniques aim at determining the usability of the web application and can be applied to a range of web applications [5]. Despite of so many different techniques and models being developed, the factors affecting the UX have not been reviewed formally. Additionally, regardless of multiple techniques undertaking the qualitative experiences of users, evaluation techniques with low overhead costs are still not discovered [6].

This study majorly concentrates on developing a model that helps in evaluating the UX of web applications. The evaluation is done keeping in consideration different parameters that affect the UX. Based on the evaluation, all the parameters are rated individually. These ratings are then further used to determine the overall UX rating for the web applications. Along with the evaluation, the model also gives out suggestions that determine the shortcomings of the web application. These suggestions are guidelines to overcome those shortcomings and deliver a better UX [7].

The rest of this paper is structured as follows: Section 2 exhibits the related works. Section 3 introduces the methodology in detail. Section 4 exhibits the results of the experimental setup. Section 5 exhibits the conclusion and the future enhancements for this work.

\section{RELATED WORKS}

The following literature survey exhibits the existing works that are significant in this study based on the UX evaluation parameters and the techniques used for evaluation:

The objective of UX investigation and assessment methods or techniques is to better consumer loyalty through the utility, convenience and nature of cooperation with applications, administrations and services. So far UX, bargains more with the assessment of momentary objectives and subsequently on aspects identifying with the underlying appropriation of new applications plans. However, the applications advance overlong length of time also, therefore new strategies should be found to perform UX assessment. Typically, evaluation of UX is quite tough, given there are a variety of components that affect the same. In order to make the assessment organized and structured it is very necessary to elucidate the components and determine a test plan. Evaluation methods Repertory Grid Technique (RGT) and UX Curve helps in determining these components and graphs to evaluate UX. Nevertheless, these methods are effective but other assessment methods are required to evaluate UX efficiently [8].

UX has developed and tended to issues of plan and assessment for collaboration between clients, interfaces and PCs past the level of ease of use. The advancement, what's more, enthusiasm for UX rises fundamentally as the necessities of clients turn out to be more intricate and enhance. UX is progressing in HCI which incorporates numerous interconnected gadgets, new gadget plans, social needs, for example, versatile, omnipresent, social and unmistakable figuring advances. UX has moreover gone past conventional usability building. It incorporates clients' emotions, inspirations and qualities which are given more accentuation notwithstanding the proficiency, adequacy and essential fulfilment which characterized the "customary" which means ease of use. UX covers the product advancement process, site, what's more, item outline; it is in this way critical to join the enthusiasm of various partners. The field of UX is normal to cover all the encompassing point of view on how a man or client feels about utilizing a framework or item. The emphasis is on delight and qualities as opposed to on execution. The examination finds that the sort and improvement stage classifications are most mainstream (i.e. they have the best recurrence) and consequently have the greatest effect. This is since, in view of these classifications, UX analysts and professionals can undoubtedly distinguish the most reasonable UX evaluation methods for their exploration [9].

There are several assessment strategies that help designers in the making of intelligent electronic items, administrations and situations that are both simple and suitable to use for the target audience. This target audience can be the widest scope of individuals, comprising individuals with learning difficulties or a specific group of people [10]. Issues in the domain of convenience might be enhanced in UX as a result of its bigger degree. The primary issues are definition, demonstrating, technique determination and transaction between assessment and advancement. It is expected to build up a more profound comprehension according to what the assessment input constitutes programming advancement, particularly qualities like fun, trust, tasteful esteems are concerned [11]. A similar strategy presents UX as model framed using components that exist within 5 planes of the development process namely strategy, scope, structure, skeleton and surface. 
This model helps in identifying how software interface elements and hypertext theory elements interact with one another [12].

Ease of use and UX are critical metrics for inspecting the quality of a web application. The difficulties confronted in manual ease of use testing offered to ascend to the development of ease of use evaluation instruments in the market. As these instruments might be exorbitant, it is imperative for ease of use specialists to legitimize the advantages and economics of these instruments. Thus, the aim is to assemble the prerequisites for web application UX testing which is trailed by building up an Open Source Software (OSS) called Ultimate Dependable and Native Usability System (URANUS) to help majority of the procedures utilized for ease of use test. The final aim is to standardize URANUS against existing devices. The procedures utilized amid prerequisites gathering included writing audit and meetings with the target group of onlookers trailed by the advancement of URANUS utilizing the Rapid Application Development (RAD). In light of the LBUT and RUT correlations, URANUS ended up scoring relatively high as far as advantages with ease of proprietorship.

The improvement of URANUS depends on the prerequisites got from the experts and by benchmarking against existing instruments. In spite of the fact that all necessities were finished, an examination with former UX instruments demonstrates that URANUS has a moderately decent adjust scorecard of $65 \%$ contrasted with Morae and Tobii Studio that has $70 \%$ what's more, $65 \%$ individually. This demonstrates URANUS can be utilized dependably and will profit ease of use specialists. What's more, the TCO and COO examination performed utilizing both contextual investigations demonstrates that URANUS has the most minimal expense of proprietorship and expense of operations. This demonstrates that URANUS requires the minimum budgetary speculation contrasted with the other financially accessible devices [13].

\section{METHODOLOGY}

The primary focus of this study is to develop a mechanism or a system which analyses a web application based on some predefined UX parameters. These parameters will be used as the evaluation criteria for the overall UX of the web application by determining a rating for each of the parameters which will, in turn, determine the overall rating of the web application. The whole process flow of the proposed model is presented in Figure 1.

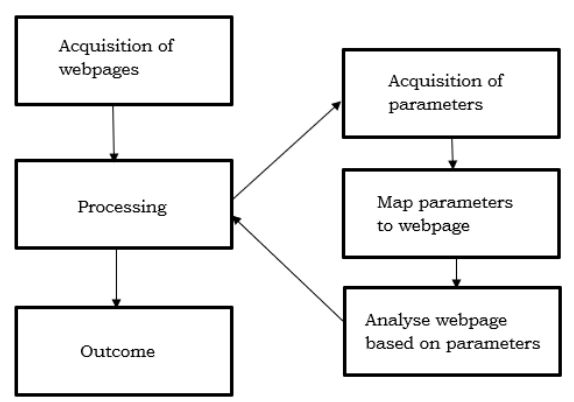

Figure 1. Process flow of proposed model

The first step is to acquire the webpages/website/web application that needs to be analysed. These web pages act as an input to the system. After the input acquisition phase, comes the processing phase. The processing phase has sub-activities: First, the parameters are to be acquired based on which the webpages will be evaluated. There are different categories of the parameters like - web performance optimization, look and feel, responsive web design, etc. Once the parameters are acquired those parameters need to be mapped to the webpages in order to analyse the webpages. Then, to quantify the UX of the selected webpage the parameters are analysed. Finally, after analysing the parameters the final output is received in the form of recommendations and suggestions that can help improve the UX.

The proposed system will be using the web performance optimization parameters. These parameters focus on the best practices and techniques implemented to improve website performance and display contents faster to the end-user, as well as save bandwidth and energy power. Optimized content efficiency/data delivery and improved server response time. This experimental study has been divided into two phases: Phase 1 discloses the identification of the parameters and Phase 2 exhibits the computation of the identified parameters. 


\subsection{Phase 1: identification of the parameters}

Before starting the evaluation of UX with any kind of methodologies and techniques, it is very important to understand, "What does UX depend on?", "What factors influence UX?", "What are the parameters that affect the overall UX?", etc. The answer to these questions can give a complete clarity on how to get started with the evaluation or assessment. So, for assessing UX of a web application, it is very crucial to understand the factors or parameters that impact UX of web application.

There are a number of parameters that impact UX of web applications in various ways. These parameters can be broadly classified into two sets: Technical Parameters and Non-Technical Parameters. The technical parameters portray the technical aspects of a web application such as response time of the web application, number of HTTP requests made to load a particular webpage, number of empty references in HTML tags, number of redirects, etc. On the contrary, the non-technical parameters deal with the visual appearance of the web application like design, layouts, background, colours, etc. In other words, we can say that the technical parameters determine the technicalities of the web application while nontechnical parameters determine the look and feel of the web application. The design is important, but however, a good visual design alone cannot necessarily provide a good UX. For instance, a great visual design cannot save a web application that is too complex to use. So, for providing optimal UX, it is necessary to have a balance of both technical and non-technical parameters.

The objective of Phase 1 was to identify a set of UX parameters that would be used for evaluating the UX of a web application. According to a model proposed by [14] which is based on Herzberg's twofactor theory, the design factors for a web application can be classified into two categories: hygiene and motivator. All the factors whose existence makes the web application usable or operative and valuable, while unavailability of these factors generates discontent and displeasure in users are known as hygiene factors. An example of this would be hyperlinks on a webpage, i.e. live hyperlinks make the web application operational but broken hyperlinks create displeasure and frustration in users. On the other side, the factors which add extra value to the web application or enhance it by impacting the user satisfaction are known as motivators. Lack of motivators doesn't affect the UX much, considering that the hygiene factors are in order. The simplest example of motivators is the use of multimedia in web applications, i.e. presence of multimedia enhances the UX. In a similar line of thinking, [15] have also defined certain criteria to evaluate UX such as visual hierarchy, contrast, use of colour, labels, feedback, forms, information scalability, physical constraints, first-time user and screen size. These criteria focus only on the visual appearance of the web application and not the technical details.

Along with the above-mentioned criteria and different parameters, there were furthermore different parameters that were studied. From these different sets of parameters, a final set of UX parameters consisting of technical and non-technical parameters was identified for evaluating UX of a web application. The final set of parameters are presented in Table 1.

Table 1. Identified parameters

\begin{tabular}{lll}
\hline \multicolumn{1}{c}{ Parameter } & \multicolumn{1}{c}{ Type } & \multicolumn{1}{c}{ Description } \\
\hline Response Time & Technical & It determines the time required to load the web application \\
Responsive Web Design & Non-Technical & It determines how well the web application design adapts to different screen sizes \\
HTTP Requests & Technical & It determines the minimum number of requests to load the web application \\
Request Size & Technical & It determines the size of the HTTP request \\
Empty SRC or HREF & Non-Technical & It determines the number of empty sources or hyper references in HTML tags \\
Images without ALT attribute & Non-Technical & It determines the number of image tags without ALT attribute \\
Misplaced CSS & Technical & It determines the number of misplaced CSS files \\
Misplaced JS & Technical & It determines the number of misplaced JS files \\
Internal CSS & Non-Technical & It determines the number internal CSS files \\
\hline
\end{tabular}

\subsection{Phase 2: computation of the parameters}

The objective of Phase 2 was to compute the set of technical and non-technical parameters that were identified in Phase 1. The proposed model or system that evaluates the UX of web application is itself a web application designed and developed in Python. The web application is based on MVT (Model View Template) architecture. The model defines the backend of the web application, the view consists of the business logic and template defines the front end. The web application uses MySQL for the backend database to store the ratings of each parameter and the overall rating of web application. Business logic is written in Python, while the front end is developed using Django framework in Python.

The proposed system accepts the URL of a web application as an input and evaluates UX of the same by computing the ratings of technical and non-technical parameters identified in the previous phase. Based on the overall rating of the web application and ratings of individual parameters, some suggestions are given in order to improve UX of the web application. Several different techniques are used to compute the 
parameters. For instance, the response time of the web application is computed by getting the starting time and ending time of the request. Here, starting time is the amount of time (in seconds) when the request for the webpage is made and ending time is the amount of time (in seconds) when the request is served or the webpage is loaded completely. The response time is then determined by finding the difference between the starting and ending time.

Similarly, for computing the non-technical parameters like images without alternate text or number of internal CSS files used, the technique used is known as web scraping. Web scraping (also known as web harvesting or web data extraction) is a technique which is used to extract data from webpages and web applications [16]. It uses HTTP (Hyper Text Transfer Protocol) or web browsers to access the data from the webpages. The proposed model uses BeautifulSoup [17], a Python-based library which is used to parse HTML and XML documents. In order to perform web scraping, it creates a parse tree of all the parsed webpages. BeautifulSoup provides with methods that are used to find, traverse and alter the parse tree to extract the required data from the webpages.

\section{EXPERIMENTAL SETUP AND RESULTS DISCUSSION}

After the development of the proposed model, it was tested with various web applications available on the Internet to evaluate the UX of the same. The result of it was an overall rating of UX of the web application along with individual ratings of the UX parameters and a set of recommendations and suggestions based on these ratings in order to improve UX. Below is an example of StackOverflow. Figure 2 shows the homepage of the proposed model. It consists of a text field which takes Uniform Resource Locator (URL) of webpages and web applications whose UX is to be evaluated as an input. Figure 3 shows the inputted URL in the text field, i.e. https://stackoverflow.com/. Any web application that has been hosted on the Internet can be used as an input for the evaluation. On clicking the Evaluate button, it will evaluate UX of the web application whose URL is specified in the text field, in this case, StackOverFlow.

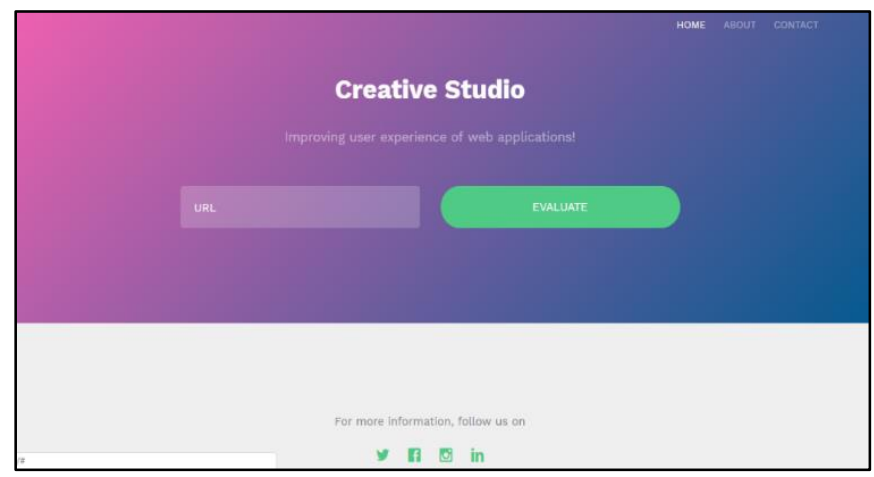

Figure 2. Homepage

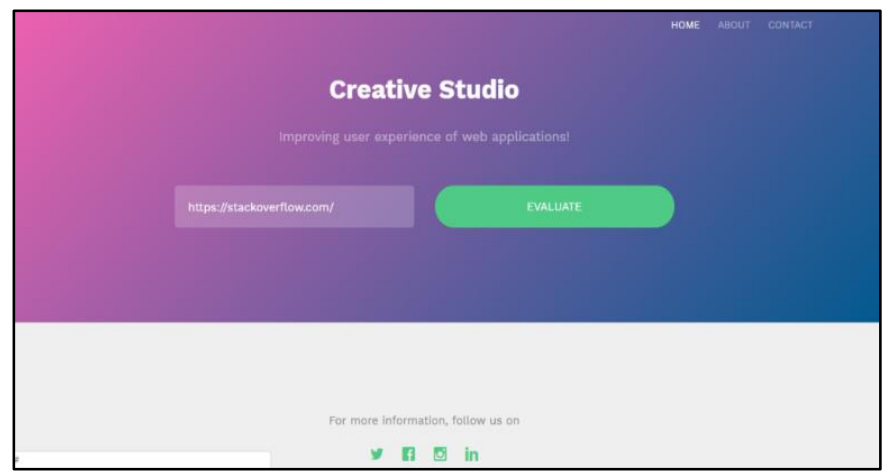

Figure 3. URL Input 
Further in the process, when the Evaluate button is clicked the system redirects to the next webpage, which displays the overall rating of the specified web application and suggestions or the recommendations for improving UX, which is presented in Figure 4. It also displays the individual ratings of each parameter along with the above-mentioned content, which is presented in Figure 5.

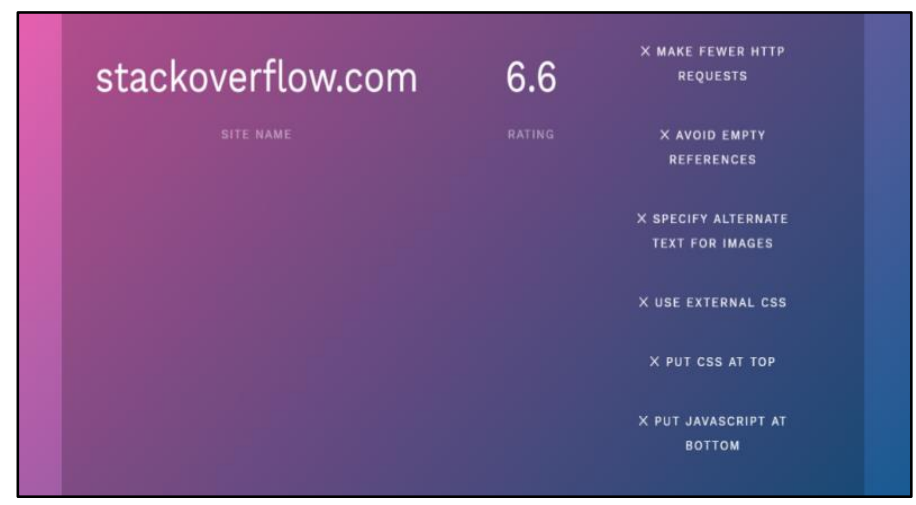

Figure 4. Overall rating \& recommendations

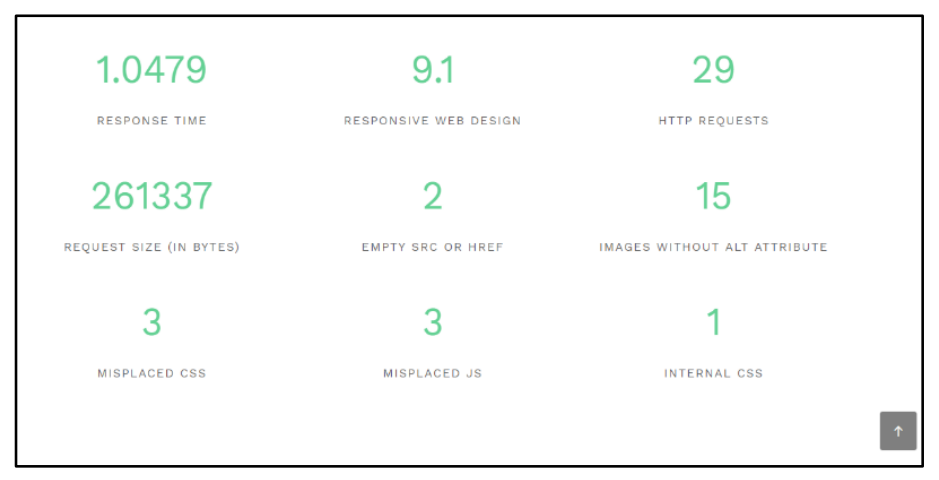

Figure 5. Detailing of the parameters

\section{CONCLUSION}

The main focus of this research is to evaluate UX of web applications based on important predefined UX parameters. Typically, the task of evaluating UX is not easy as there are several types of different parameters which impact or affect UX of web applications. For the evaluation, it is very important to identify the appropriate parameters. This, in turn, helps in making the evaluation organized and systematic.

This research identified different UX parameters consisting of technical and non-technical parameters that affected UX of web applications. All of these identified parameters were then computed based on different techniques and were given ratings. These individual ratings of parameters were combined to determine the overall rating of the web application. Results of the experimental setup show that inputting the URL of a web application induced in different ratings of each of the parameters and overall UX rating of the web application. Also, based on these ratings suggestions and recommendations were provided for improving UX of the web application. This research can be extended by adding more parameters in order to achieve better User Experience.

\section{REFERENCES}

[1] R. Hartson and P. S. Pyla, "The UX Book: Process and Guidelines for Ensuring a Quality User Experience," Morgan Kaufmann Publishers, 2012.

[2] M. Hassenzahl and N. Tractinsky, "User Experience - a research agenda," Behaviour and Information Technology, vol/issue: 25(2), pp. 91-97, 2006. 
[3] D. Sasongko, et al., "The Development of Digital Library User Interface by Using Responsive Web Design and User Experience," Indonesian Journal of Electrical Engineering and Computer Science, vol/issue: 4(1), pp. 195-202, 2016.

[4] Katie L., et al., "Categorisation of visualisation methods to support the design of human-computer interaction systems," Applied Ergonomics, vol. 55, pp. 85-107, 2016.

[5] M. F. Ukrit, et al., "Location Based Services with Location Centric Profiles," International Journal of Electrical and Computer Engineering, vol/issue: 6(6), pp. 3001-3005, 2016.

[6] N. Đ. đević, "Evaluation of the usability of Web-based applications," Vojnotehnički glasnik / Military Technical Courier, vol/issue: 65(3), pp. 785-802, 2017.

[7] K. V. V. Mattila and M. Wäljas, "Evaluating User Experience of Cross Platform Web Services with Heuristic Evaluation Method," International Journal of Art and Technology, vol/issue: 3 (4), pp. 402-421, 2010.

[8] V. Balasubramoniam and N. Tungatkar, "Study of User Experience (UX) and UX Evaluation methods," International Journal of Advanced Research in Computer Engineering \& Technology (IJARCET), vol/issue: 2(3), pp. 1214-1219, 2013

[9] S. Rajeshkumar, et al., "Taxonomies of User Experience (UX) Evaluation Methods," 3rd International Conference on Research and Innovation in Information Systems, pp. 533-538, 2013.

[10] H. Petrie and N. Bevan, "The evaluation of accessibility, usability and user experience," The Universal Access Handbook, CRC Press, pp. 10-20, 2009.

[11] E. Law and S. Abrahão, "Interplay between User Experience (UX) evaluation and system development," International Journal of Human-Computer Studies, vol/issue: 72(6), pp. 523-525, 2014.

[12] J. J. Garret, "The Elements of User Experience: User-centered Design for the Web," New Riders Publication, 2003.

[13] A. Sivaji and S. T. Soo, "Website User Experience (UX) Testing Tool Development using Open Source Software (OSS)," Proceedings of Southeast Asian Network of Ergonomics Societies Conference (SEANES), 2012.

[14] P. Zhang and M. G. von Dran, "Satisfiers and Dissatisfiers: A Two-Factor Model for Website Design and Evaluation," Journal of the American Society for Information Science, vol/issue: 51(14), pp. 1253-1268, 2000.

[15] "UX Evaluation Criteria - UXChallenge.org," UXChallenge.org, 2018. Available: http://uxchallenge.org/userexperience-ux-evaluation-criteria.

[16] “Web scraping," en.wikipedia.org, 2018. Available: https://en.wikipedia.org/wiki/Web_scraping.

[17] "Beautiful Soup Documentation - Beautiful Soup 4.4.0 documentation," Crummy.com, 2018. Available: https://www.crummy.com/software/BeautifulSoup/bs4/doc/.

\section{BIOGRAPHIES OF AUTHORS}

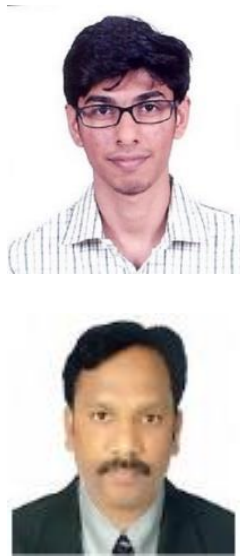

Anish Mistry is currently pursuing MCA from Department of Computer Science, CHRIST (Deemed to be University), Bengaluru, India. As part of the curriculum, he has undertaken the research and his areas of interest includes User Experience (UX), Agile Technologies and Cloud Computing.

R. Arokia Paul Rajan is currently working as Associate Professor, Department of Computer Science, CHRIST (Deemed to be University), Bengaluru, India. He holds Ph.D. in Computer Science \& Engineering from Pondicherry University, India. His research area is data management in Cloud architectures. He published 13 research papers in various international journals and conferences. 